\title{
Bariatric endoscopy: current primary therapies and endoscopic management of complications and other related conditions
}

\author{
Melissa Castro, Alfredo Daniel Guerron \\ Department of Surgery, Duke University Health System, Durham, NC 27704, USA.
}

Correspondence to: Dr. Alfredo Daniel Guerron, Department of Surgery, Division of Metabolic and Weight Loss Surgery, Duke University, 407 Crutchfield St, Durham, NC 27704, USA.E-mail: alfredo.guerron-cruz@duke.edu

How to cite this article: Castro M, Guerron AD. Bariatric endoscopy: current primary therapies and endoscopic management of complications and other related conditions. Mini-invasive Surg 2020;4:47. http://dx.doi.org/10.20517/2574-1225.2020.14

\author{
Received: 26 Jan 2020 First Decision: 15 Apr 2020 Revised: 24 Jul 2020 Accepted: 27 Jul 2020 Published: 7 Aug 2020 \\ Academic Editor: Wah Yang Copy Editor: Cai-Hong Wang Production Editor: Jing Yu
}

\begin{abstract}
The steady increase in bariatric surgery has led to room for innovation. Endoscopy has become an important tool for evaluation, diagnosis, management of complications, and even for primary bariatric interventions. Leaks are the most feared complication and new endoscopic therapies have been developed such as septotomy, doublepigtail stents, and endoscopic vacuum therapy. Additionally, primary bariatric endoscopic procedures are gaining popularity and the new procedures include intragastric balloons, stoma reduction, aspiration therapy, among others. The altered anatomy and reoperation increase the risk of complications after bariatric surgery, especially when managing conditions like achalasia, gastroparesis, and cholelithiasis. Per-oral endoscopic myotomy, per-oral pyloromyotomy, and endoscopic ultrasound-guided transgastric endoscopic retrograde cholangiopancreatography provide a less invasive approach to address these conditions. This narrative review article intends to expose current endoscopic therapies for the management of primary bariatric procedures, complications and related conditions.
\end{abstract}

Keywords: Endoscopy, bariatric surgery, septotomy, leaks, endosuturing, intragastric balloons, per-oral pyloromyotomy, per-oral endoscopy myotomy

\section{INTRODUCTION}

Each year the rise of the obesity population poses a global concern, affecting more than 600 million people worldwide $^{[1]}$. Different measures have been implemented to approach this matter and bariatric surgery 
Table 1. Summary of different areas of bariatric endoscopy innovation

\begin{tabular}{|c|c|c|c|c|c|}
\hline \multicolumn{2}{|l|}{ Primary procedures } & \multicolumn{2}{|c|}{ Management of complications } & \multicolumn{2}{|c|}{ Management of concomitant conditions } \\
\hline \multirow[t]{3}{*}{ Intragastric balloons } & Orbera & Leaks & Septotomy & Achalasia & POEM \\
\hline & Reshape & & Double-pigtail stent & & \\
\hline & Obalon & & Endoscopic vacuum therapy & & \\
\hline \multirow[t]{2}{*}{ Aspiration therapy } & AspireAssist system & Weight & TORe & Gastroparesis & POP \\
\hline & & regain & ROSE & & \\
\hline \multirow{2}{*}{$\begin{array}{l}\text { Endoluminal bypass } \\
\text { liners }\end{array}$} & Duodeno-jejunal bypass sleeve & & & Cholelithiasis & EDGE procedure \\
\hline & Gastro-duodenal bypass liner & & & & \\
\hline \multicolumn{6}{|l|}{ Transpyloric shuttle } \\
\hline \multicolumn{6}{|c|}{$\begin{array}{l}\text { Magnetic compression Incisionless magnetic } \\
\text { gastrojejunostomy anastomosis system }\end{array}$} \\
\hline \multicolumn{6}{|c|}{ Mucosal resurfacing for diabetes } \\
\hline $\begin{array}{l}\text { Endoscopic sleeve } \\
\text { gastroplasty }\end{array}$ & OverStitch & & & & \\
\hline
\end{tabular}

TORe: transoral outlet reduction; POEM: per-oral endoscopy myotomy; POP: per-oral pyloromyotomy; EDGE: endoscopic ultrasoundguided transgastric endoscopic retrograde cholangiopancreatography; ROSE: restorative obesity surgery, endolumenal

remains the most effective treatment for sustained weight loss and improvement of comorbidities ${ }^{[2]}$. The American Society for Metabolic and Bariatric Surgery (ASMBS) reported 252,000 bariatric surgeries performed in 2018, an increase of 24,000 cases as compared to $2017^{[3]}$. The steady increase of bariatric procedures each year has led to room for innovation. Angrisani et al. ${ }^{[4]}$ reported that $4 \%$ of bariatric procedures corresponded to endoluminal procedures but this percentage may be underestimated. Endoscopy has become an important tool for evaluation, diagnosis, management of complications, and even as primary bariatric interventions. Besides gastrointestinal specialists, advanced endoscopic procedures can be additionally performed by bariatric surgeons who have the knowledge and skills to perform them. Nonetheless, it is important to emphasize that management of these patients must be done in a multidisciplinary approach with enough expertise to handle these cases, which includes participation of both the bariatric and gastrointestinal specialists.

Endoscopy can be applied in various ways in bariatrics, including preoperative evaluation to study the anatomy, preoperative planning for revisions, intraoperative management to address inadvertent technical errors, postoperative management for complications, primary bariatric procedures, among other applications. With the evolution of minimally invasive techniques, endoscopy stands as an attractive alternative for the management of obesity. Bariatric endoscopy is an essential tool in the armamentarium of surgeons dedicated to the management of morbid obesity. These less-invasive endoscopic techniques serve as a promising alternative for the management of bariatric patients.

\section{PRIMARY PROCEDURES}

Bariatric surgery stands as the most effective therapy for sustained weight loss and improvement of comorbidities $^{[5,6]}$. The ever-rising epidemic of obesity has led physicians to develop non-surgical alternatives for the management of these patients. Endoscopic management of obese patients has several benefits over bariatric surgery such as the less-invasive nature of the procedures and fewer complications. A summary of available endoscopic therapies can be found in Table 1. Additionally, endoscopic techniques give the opportunity to patients who are not eligible for surgery or who prefer a less-invasive approach.

\section{Intragastric balloons}

Intragastric balloons (IGB) were first used in 1982 with the purpose of inducing a sense of satiety by a space-occupying device ${ }^{[7]}$. Various types of IGB have been developed; however, only three of them are FDA approved, the Orbera, ReShape, and the Obalon $\mathrm{IGB}^{[8]}$. The most common intragastric balloon used worldwide is the Bioenterics Intragastric Balloon which is made of silicone-based material and filled with 
saline or air that can hold up to 400 to $800 \mathrm{~mL}^{[7]}$. In the United States, it is sold as the Orbera Intragastric Balloon System (Apollo Endosurgery, Austin, TX), which was approved for use in $2015^{[9]}$. Other available balloons include ReShape (ReShape Medical Inc., San Clemente, CA) that consists of a dual balloon system. The Obalon (Obalon Therapeutics, San Diego, CA) is distinguished from the others because it is placed in the stomach by swallowing a deflated balloon in the form of a capsule, thus having a smaller capacity $(250 \mathrm{~mL})^{[9]}$.

A study evaluated the use of the Orbera intragastric balloon alone and before definite bariatric surgery over a period of 16 years. The authors reported positive short-term outcomes for the use of IGB alone, with EWL of $17.2 \%$ at 1 year; however, after 2 years weight loss was not maintained. The patients who had placement of the IGB and then underwent bariatric surgery had long-term sustained weight loss. The authors concluded that IGB should be used as a bridge therapy to definitive therapy ${ }^{[10]}$. Moore et al. ${ }^{[11]}$ evaluated the outcomes at 6 months in 1,343 patients who had one or up to three Obalon IGB placed. The majority of adverse events were mild and did not require intervention; nonetheless, two severe adverse events were reported which included balloon slippage to the pylorus and gastric perforation. Although weight loss was achieved in the population studied, long-term data are still needed to prove its efficiency. The most common adverse events reported with the use of IGB are abdominal pain, nausea, vomiting, and balloon deflation ${ }^{[10-12]}$. The use of IGB should be considered as either bridge therapy to definitive bariatric surgery or in patients who need only moderate weight loss in combination with behavior modification.

\section{Aspiration therapy}

Aspiration therapy removes up to $30 \%$ of gastric contents after a meal through a percutaneous endoscopic gastrostomy tube thus reducing the amount of chyme that reaches the small bowel for absorption ${ }^{[13,14]}$. It should be considered in cases of severe obesity as a bridge therapy to more effective weight loss procedures. The AspireAssist System (AspireAssist; Aspire Bariatrics, King of Prussia, PA) has two components, the A-Tube and the skin-port that is attached to the tube and in the US is approved for patients with BMI of 35 to $55 \mathrm{~kg} / \mathrm{m}^{2}$ who have previously failed to lose weight with non-surgical alternatives. Four studies have been conducted to evaluate the effects of aspiration therapy on weight loss ${ }^{[13]}$. A US pilot study comprised of 18 obese patients, compared weight loss outcomes in patients with aspiration therapy $(n=11)$ and patients with lifestyle therapy $(n=7)$ at 1 year. The aspiration therapy group lost $18.6 \% \pm 2.3 \%$ of their body weight versus $5.9 \% \pm 5.0 \%$ in the lifestyle therapy group ${ }^{[15]}$. A multi-center, randomized, controlled trial, the PATHWAY trial, evaluated 1-year outcomes in 207 patients who had the aspiration system (AspireAssist System) placed compared to patients who had lifestyle counseling alone. The authors reported 37.2\% EWL in the AspireAssist System group and 13.0\% EWL in the lifestyle counseling group. Additionally, the Impact of Weight on Quality of Life score had a higher increase in the treated group across all five score measures. The majority of adverse events occurred within 7 days of the procedure and included peristomal granulation tissue, abdominal pain, nausea/vomiting, and other less infrequent events. Five serious adverse events were reported in 4 patients and consisted of peritonitis, severe abdominal pain, pre-pyloric ulcer, and A-tube replacement because of skin-port malfunction ${ }^{[16]}$. A multicenter study conducted in Europe included 201 participants and followed them at 1, 2, 3, and 4 years after the procedure. The authors reported reduction in weight, glycated hemoglobin, triglycerides, and blood pressure. There were serious complications that included buried bumpers in 7 participants which resolved by replacement/removal of the A-Tube, and one case of peritonitis that resolved with antibiotic treatment ${ }^{[17]}$. Although preliminary results seem promising, this device can't be applied alone for obesity management which makes it a less attractive alternative.

\section{Endoluminal bypass liners}

There are two endoluminal bypass liners that are still being trialed and yet to be FDA-approved, the gastroduodenal bypass liner and the duodeno-jejunal bypass sleeve. Both systems create a mechanical barrier between food and the proximal small bowel, which mimic the excluded biliopancreatic limb of a Roux- 
en-Y gastric bypass (RYGB) ${ }^{[14,18]}$. The most studied of these devices is the duodeno-jejunal bypass sleeve known as the Endobarrier (GI Dynamics, Boston, MA) which is removed endoscopically 12 months after placement ${ }^{[19]}$. Several trials have demonstrated the potential benefit of the Endobarrier for weight loss and improved glucose control. A pilot study comprised 12 patients, reported a mean \%EWL of $23.6 \%$ in 12 weeks and only two patients required removal of the Endobarrier due to inappropriate device placement. Of the 12 patients included, four were diabetic and did not require their diabetes medications during the time the device was placed ${ }^{[20]}$. A prospective trial of 42 subjects reported that the device was successfully implanted in 39 patients and they were followed up for 1 year. They reported a $19.9 \% \pm 1.8 \%$ reduction of total body weight loss and the EWL was $47.0 \% \pm 4.4 \%{ }^{[21]}$. Although some studies have reported positive outcomes with this device, others have reported various adverse effects ${ }^{[7,14]}$. The ValenTx (ValenTx, Inc. Carpinteria, CA, USA) is a gastro-duodenal bypass liner that is still being studied and little data exists regarding long-term outcomes. A study involving 12 patients who had the ValenTx device placed for 1 year demonstrated a mean percentage EWL of $54 \%$ but only 6 of the patients had a fully attached and functional device $^{[22]}$. Further studies and improvement of the device are still needed for the device to be approved and considered as an option for obesity.

\section{Transpyloric shuttle}

The Transpyloric Shuttle (TPS) (BARONova Inc, San Carlos, CA) is a large spherical bulb attached to a smaller cylindrical bulb through a catheter that results in delayed gastric emptying and is FDA approved. It is delivered transorally in the stomach and once it has been deployed, gastric and intestinal contractions pull the TPS into the duodenum which stops at the pylorus, causing intermittent gastric outlet obstruction and thus, delaying gastric emptying. Few studies have evaluated its safety and effectiveness. A singlecenter prospective, non-randomized trial of 20 patients with a mean BMI of $36.0 \mathrm{~kg} / \mathrm{m}^{2}$ reported a mean EWL of $31.3 \% \pm 15.7 \%$ at 3 months and a mean EWL of $50.0 \% \pm 26.4 \%$ at 6 months. The device had to be removed in two cases due to persistent gastric ulceration ${ }^{[23]}$. A multicenter randomized sham-controlled trial in the US evaluated the safety and effectiveness of the TPS for weight loss. All patients $(n=203)$ who were treated with TPS reported an adverse event and 10/203 patients presented with a serious adverse event, no mortality was reported ${ }^{[24]}$. These results have not yet been published but the preliminary results demonstrate that there is still a need for improvement so that it can be safely used in obese patients.

\section{Magnetic compression gastrojejunostomy}

Magnetic surgery for gastrointestinal surgery is an appealing approach that offers promising results ${ }^{[25]}$. The Incisionless Magnetic Anastomosis System (GI Windows, Boston, MA) creates an intestinal bypass through compression of self-assembling magnets delivered endoscopically. The magnets are deployed in the proximal jejunum and in the ileum with the use of pediatric colonoscopes under fluoroscopic visualization. Once the magnets are deployed, they will couple and cause necrosis in the tissue, which leads to the formation of an anastomosis and a dual pathway. The magnets will be expelled naturally after a couple of days. Ryou et al. ${ }^{[26]}$ evaluated the feasibility of this device on eight pigs. By day 10, the anastomosis had already been formed and by day 90, the magnets had been completely expelled with full anastomotic patency. The first human pilot study included ten patients and used laparoscopy to confirm adequate magnet coupling. The anastomosis was created in all subjects and no device-related serious adverse events were reported. Patency of the anastomosis was confirmed at 2, 6, and 12 months. The mean total weight loss was $14.6 \%$ and the mean excess weight loss was $40.2 \%$ at 12 months. This study also showed a decrease in Hemoglobin $A_{1 C}$ and fasting glucose in diabetic patients ${ }^{[27]}$. Even though these results sound promising, further studies and longer-term results are needed to confirm the utility of the incisionless anastomotic system.

\section{Mucosal resurfacing for diabetes}

Duodenal mucosal resurfacing (DMR) is a procedure that consists of hydrothermal ablation of the duodenal mucosa. Once the catheter is advanced into the duodenum, a balloon is inflated with heated 


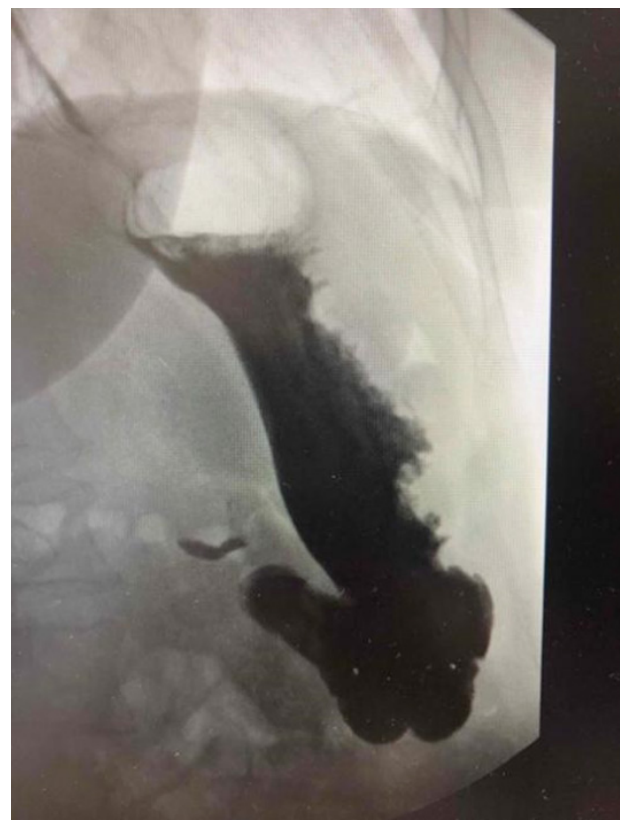

Figure 1. Contrast image of post endoscopic gastroplasty

water to ablate the duodenal mucosa circumferentially. This therapy has shown positive outcomes in management for diabetes ${ }^{[14,28,29]}$. The hypothesis behind it is that through duodenal mucosal ablation, there will re-epithelialization with normal mucosa ${ }^{[14]}$. A prospective multicenter trial showed sustained improvement of glycated hemoglobin (HbA1c) at 12 months in 37 patients with type 2 diabetes (T2D) who underwent $\mathrm{DMR}^{[28]}$. DMR has shown improvement in glycaemic control in patients with $\mathrm{T} 2 \mathrm{D}$; however, the mechanisms of how glycemic control is achieved are still under study.

\section{Endoscopic sleeve gastroplasty}

The endoscopic sleeve gastroplasty is a procedure whose technique has been modified to achieve better results. The procedure is done with the use of The Overstitch (Apollo Endosurgery, Austin, TX) suturing device which was recently approved by the FDA. The procedure consists of the placement of transmural sutures in a triangular fashion such that it creates a tubular shape similar to sleeve gastrectomy [Figure 1$]^{[14,30]}$. A prospective study that included 154 patients, evaluated total body weight loss (TBWL) at 1, 3, 6, 12 and 24 months after endoscopic gastroplasty using The Overstitch (Apollo Endosurgery, Austin, TX). At 2 years of follow-up, $85.7 \%$ of patients surpassed the threshold of $25 \% \mathrm{EWL}$, suggested by the American Society for Gastrointestinal Endoscopy (ASGE) and the ASMBS ${ }^{[30]}, 193$ patients from 7 centers underwent endoscopic sleeve gastrectomy using The Overstitch device and were followed up at 6 months and 1 year after the procedure. There was a BMI decrease of 5 and 6 points at 6 months and 1 year, respectively. Most adverse events were mild and included nausea and emesis. Two severe adverse events were reported that required surgical intervention, one patient presented with a perigastric hematoma 1 week after the procedure and the second patient was found to have a leak 3 days after the procedure ${ }^{[31]}$. As anatomy is not altered with this procedure, it allows for reintervention if required. This procedure has proven to be feasible with positive long-term outcomes ${ }^{[30,31]}$; however, there has yet to be a consensus of whether this procedure should be considered over other primary bariatric procedures.

\section{INNOVATIONS IN THE MANAGEMENT OF COMPLICATIONS}

Although low in incidence, patients may present with complications after bariatric surgery. It is important to do a thorough evaluation of the patient to determine the diagnosis and advocate proper management. 


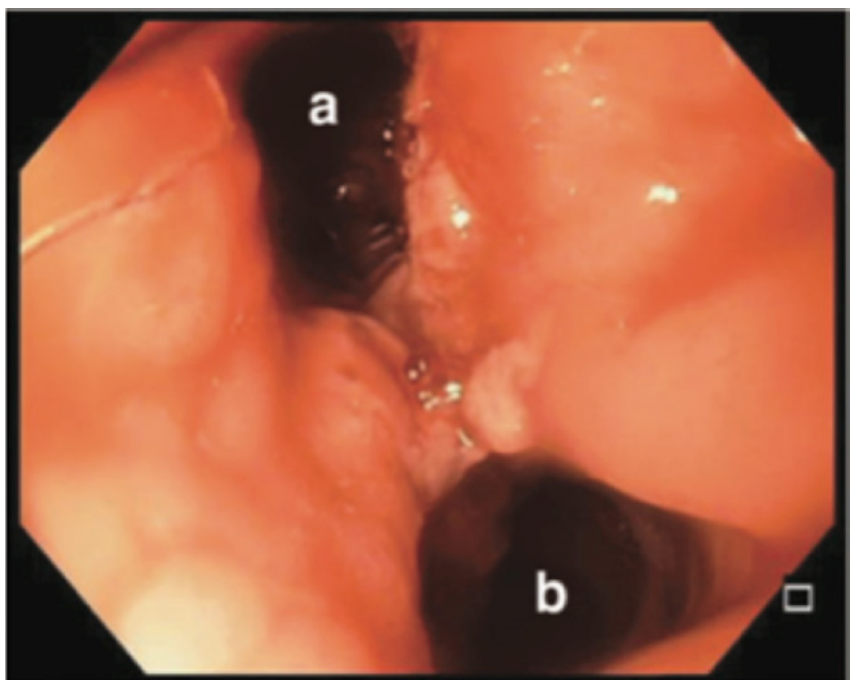

Figure 2. Endoscopic view of the septotomy completed with the abscess cavity fully exposed to the gastric lumen. (a) Abscess cavity; (b) gastric lumen

Common symptoms involve nausea, vomiting, abdominal pain, inadequate gain loss or weight regain, dysphagia, dyspepsia, reflux, increased stools, among others ${ }^{[32,33]}$. Complications may present in the early or late postoperative period. They can present as hemorrhage, leaks, fistulas, strictures, ulcers, or erosion and their management will depend according to the type of complication. When the patients' condition is suitable, less invasive techniques are preferred. Many endoscopic procedures have been widely used over the years; however, newer devices have been recently developed.

\section{Leaks}

One of the most feared complications of bariatric surgery is the development of gastric leaks and fistulas. Even though leaks have a low incidence rate, their presentation causes a 2 -fold increase in mortality and a 6 -fold increase in hospital stay ${ }^{[34]}$. In RYGB patients, most leaks arise at the gastrojejunal anastomosis, whereas in laparoscopic sleeve gastrectomy (LSG), they are usually found along the staple line $e^{[35]}$ and at the gastroesophageal junction ${ }^{[36-38]}$. Leaks can be classified according to their time of presentation as acute, early, late, or chronic; presenting $<1,1-6,6-12,>12$ weeks after surgery, respectively ${ }^{[39]}$. Endoscopic treatment with the use of stents, sealants, or clips has been broadly used in bariatric surgery with positive outcomes described in the literature ${ }^{[40,41]}$. Of these, the most common used are stents. New and innovative endoscopic procedures are now available and suppose a promising alternative.

\section{Septotomy}

Abscess septotomy is a procedure utilize to control late to chronic leaks, that consists in dividing the septum formed between the abscess cavity and the gastric lumen. This allows for equalization of pressures in both cavities, favoring the drainage of the abscess cavity into the sleeve lumen [Figure 2]. Ortega et al. ${ }^{[37]}$ reported their experience with chronic leaks after LSG that were managed successfully with abscess septotomy in combination with aggressive dilation of the sleeve and axis rectification in order to promote distal drainage and improved management of the intraluminal pressures. Shnell et al ${ }^{[39]}$ reported 10 patients with late and chronic leaks that were also effectively managed with septotomy. The authors performed on average 5 endoscopic sessions to completely resolve the leak. Nonetheless, two cases with a small perigastric cavity $(<15 \mathrm{~mm}$ ), only needed one session to achieve leak resolution. They consider several sessions necessary to adequately drain the abscess cavity, as well as performing stricture dilation for better outcomes. This procedure represents a safe, feasible, and less invasive approach that should be strongly considered for the management of late and chronic postoperative leaks ${ }^{[42-45]}$. In our center, we prefer 
septotomy in combination with aggressive axis rectification using achalasia balloons. Septotomy procedures have better results in abscess with larger cavities. For linear abscess in which a septum dividing the lumen and the abscess cavity is small or minimal, we opt to use a pigtail catheter to control the abscess with endoluminal drainage. Unfortunately, some cases end up in esophagojejunostomy operations.

\section{Double-Pigtail stent}

Another technique available for late and chronic leaks that has gained more popularity over the recent years due to its safety, efficacy, lower cost, and good tolerance is the use of double pigtail stents. The procedure is performed by advancing a guidewire into the communicating collection under fluoroscopic guidance. Once the guidewire is in place, a double-pigtail stent is placed, which allows drainage of the abscess into the gastric cavity ${ }^{[24]}$. A systematic review included 385 patients with gastric leak after sleeve gastrectomy that were treated with double-pigtail stents as a primary or secondary procedure. The success rate of leak resolution by using the double-pigtail stent as a first-line therapy or as a rescue therapy was $84.71 \%$ and $78.05 \%$, respectively. The study also reported a complication rate of $13.73 \%$, the most common being drainage migration. Furthermore, the authors conclude that this technique has proven to be efficient and well-tolerated, with the additional benefit of reducing costs by having a shorter length of stay ${ }^{[2]]}$.

\section{Endoscopic vacuum therapy}

Endoscopic vacuum therapy (EVT) is a technique that is increasingly used among surgeons and endoscopists to treat leaks. The procedure consists of the placement of a sponge drainage system into the perigastric cavity, which drains the content of the leak by applying negative pressure. This system can be placed intracavitary or within the stomach lumen at the entrance to the perigastric cavity ${ }^{[4,47]}$. Archid et al. ${ }^{[46]}$ reported 8 patients who developed a staple line leak following sleeve gastrectomy that were treated with EVT. The leak resolved completely in seven of the eight cases, representing an $87.5 \%$ success rate. Only one complication was reported in a patient who developed bleeding from a short gastric vessel. A study developed an online survey to evaluate the current practice of international expert therapeutic endoscopists regarding the management of upper gastrointestinal leaks. The study showed that EVT allowed for adequate drainage of the cavity and warranted granulation ${ }^{[42]}$. One major limitation of this procedure is that the sponge needs to be replaced every 3 to 5 days $^{[42,47]}$. Nonetheless, EVT is a safe and feasible approach for leak management.

\section{Weight regain}

Weight regain or insufficient weight loss can be challenging to manage and involves a thorough multifactorial and multidisciplinary evaluation. In our center we start with endoscopic and imaging (UGI) evaluation to assess for complications. In particular, we assess for gastrojejunal dilation, pouch dilation, gastro-gastro fistulae, etc. All patients undergo nutritional and psychological evaluation to modify habits and behaviors. Occasionally, pharmacotherapy is added to the treatment in order to maximize success.

There are several endoscopic options to manage weight regain. The gastrojejunal anastomosis size can be reduced in order to maximize restriction. Transoral outlet reduction (TORe), aims to reduce the size of the anastomosis by placing sutures in specific locations surrounding the anastomosis. The OverStitch (Apollo Endosurgery, Austin, TX) and the EndoCinch (Bard Davol, Murray Hill, NJ) are two devices that can be used for the TORe procedure. The OverStitch has proven to be more effective for weight loss compared to the EndoCinch and is used in a similar fashion as for endoscopic sleeve gastroplasty but following the TORe technique ${ }^{[48]}$. The first technique involved placing interrupted sutures at the gastrojejunal anastomosis and the second, the creation of a pursestring. The latter resulted in greater weight loss at 12 months compared to the traditional interrupted suture pattern $(19.8 \% \mathrm{EWL}$ with the purse-string technique vs. $11.7 \%$ EWL with the interrupted technique, $P<0.001)^{[49]}$. Various studies have demonstrated the safety and feasibility of this procedure. A recent study evaluated the amount of weight loss at 1,3 , and 
5 years after the initial TORe in 331 post-RYGB patients who had weight regain or inadequate weight loss. The results showed that TORe is a safe, effective, and durable therapy for weight regain following $\mathrm{RYGB}^{[50]}$.

Restorative Obesity Surgery, Endolumenal (ROSE) is another available option to reduce the size of the gastric pouch and the anastomosis. The procedure consists of placement of sutures that surround the anastomosis or in the stomach wall creating plications that allow for stoma reduction. A prospective multicenter study that included 116 patients, reported that ROSE was successfully performed in 112 patients and had an average of $\%$ EWL of $18 \%{ }^{[51]}$.

\section{Strictures}

Strictures after bariatric surgery represent a technical challenge. The incidence of this complication varies significantly based on different operations and different technique. Laparoscopic Roux-en-Y gastric bypass (LRYGB) has the highest incidence of anastomotic strictures ranging from $3 \%-27 \%{ }^{[52]}$ while the incidence of stenosis after LSG ranges from $0.2 \%$ to $4 \%{ }^{[53]}$. The most common technique to treat this problem is endoscopic balloon dilation.

Dilation of gastro-jejunostomy strictures with endoscopic balloons has proven to be highly successful. In a study that included sixty-one patients, all responded to dilation without need for formal surgical revision with a $2.2 \%$ incidence of perforation ${ }^{[52]}$. The technique involves proper identification of the anatomy and estimation of the narrowing. The diameter of commonly used diagnostic upper endoscopes ranges between 9 and $10 \mathrm{~mm}$. Inability to pass the scope necessitates the use of smaller balloons, typically 6 or $8 \mathrm{~mm}$. Sequential dilations can be attempted using manometric feedback. Once a maximal diameter is reached, the balloon is held in place for $1 \mathrm{~min}$. We rarely exceed a diameter of $15 \mathrm{~mm}$ after LRYGB at our institution. Long standing strictures are less likely to resolve with endoscopic dilatation and may require operative revision.

\section{MANAGEMENT OF CONCOMITANT CONDITIONS IN THE BARIATRIC PATIENT}

Like any other patient, multiple gastrointestinal conditions may arise such as achalasia, gastroparesis, and cholelithiasis; however, their management pose a challenge for the surgeon as the anatomy is altered after bariatric surgery and reoperative fields increase the risk of complications. New endoscopic have been described and are currently taking more predominance than the surgical approach.

\section{Achalasia in bariatrics}

Obesity impacts esophageal function by altering the lower esophageal sphincter resting pressures and motility. Achalasia is an uncommon disease that could also present concomitantly after bariatric surgery. Myotomy had been the preferred therapy to treat patients with achalasia ${ }^{[54]}$. Most recently, Inoue et al. ${ }^{[55]}$ introduced esophageal myotomy endoscopically instead of open surgery or laparoscopically, the procedure known as per-oral endoscopy myotomy (POEM). The therapy consists of dissection of the circular muscle bundle through a previously created submucosal tunnel at the gastroesophageal junction; after completion, the mucosal entry is closed with hemostatic clips. Symptom control after POEM is comparable to that seen with laparoscopic Heller myotomy $(\mathrm{LHM})^{[54-56]}$. If achalasia presents in post-bariatric patients, the management remains the same as with a normal anatomy patient. However, given that performing LHM would involve an additional operation and thus, potential increased operative risk, we consider POEM a better, non-surgical, less invasive alternative in this setting as well as for failed LHM where POEM serves as a feasible, safe, and minimally invasive technique ${ }^{[5,58]}$. We believe that POEM should be considered as first option for managing patients with achalasia after bariatric surgery. Recently, Sanaei et al. ${ }^{[59]}$ explored the outcomes of POEM in 10 patients with RYGB anatomy that presented with achalasia. All patients were treated successfully, with no complications, and significant symptom improvement. Luo et al. ${ }^{[60]}$ also reported a case of a 67-year-old female with previous RYGB that developed achalasia and was successfully 


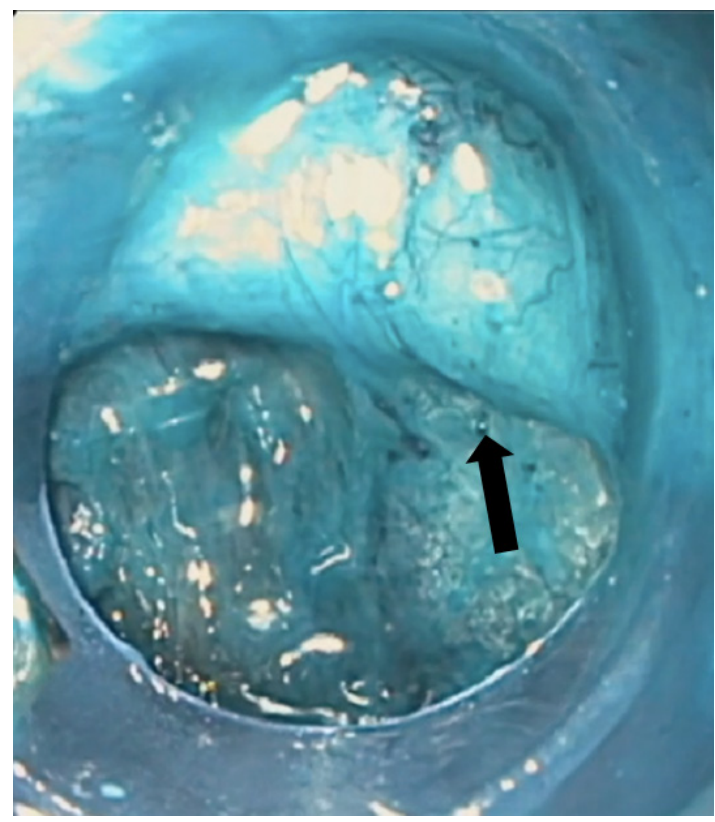

Figure 3. Endoscopic view of the pylorus. Endoscopic view of the transition between the pyloric muscle and the submucosal plain depicted by the arrow

treated with POEM. Bashir et al. ${ }^{[54]}$ described 6 patients with achalasia and surgical history of RYGB who underwent POEM; all but one patient had improvement of symptoms. While this procedure requires advanced endoscopic skills and more studies in obese patients are required, it shows to be a promising alternative for the management of achalasia in post-bariatric patients as it avoids manipulation of an already explored hiatus, providing greater benefit for the patient.

\section{Gastroparesis in bariatrics}

Gastroparesis is characterized by a delay in gastric emptying without a mechanical obstruction that includes a multifactorial etiology. Previously therapies used include botulinum toxin injection, endoscopic transpyloric stent placement and fixation, and laparoscopic pyloroplasty ${ }^{[61]}$. Endoscopic per-oral pyloromyotomy (POP), also known as gastric per-oral endoscopic myotomy (G-POEM), was recently introduced as an alternative therapy for pyloric dysfunction. A mucosal lift is performed along the lesser curve of the stomach with a regular gastroscope. With an endoscopic knife, a transverse mucostomy is made after which, a submucosal tunnel is developed using the same instrument. The pylorus is then identified and divided completely [Figure 3]. Once finished, the mucostomy is closed with several endoscopic clips ${ }^{[62]}$. It is worth mention that this procedure is more technically demanding and requires advanced endoscopic skills to perform. Rodriguez et al. ${ }^{[63]}$ assessed 100 patients with refractory gastroparesis that were treated with POP. Preoperatively, the mean BMI was $25.3 \mathrm{~kg} / \mathrm{m}^{2}$. Of those, $21 \%$ of the patients had a BMI $>30 \mathrm{~kg} / \mathrm{m}^{2}$. There was improvement of the Gastroparesis Cardinal Symptom Index (GCSI) score on all types of gastroparesis. Complications occurred in $10 \%$ of the patients including gastrointestinal bleeding, dehydration, capnoperitoneum, and subcutaneous emphysema. Farha et al. ${ }^{[64]}$ reported a case of a 43-year-old female with a history of LSG who presented with upper gastrointestinal obstructive symptoms that worsened progressively. After not responding to medical therapy or endoscopic pneumatic balloon dilation, the physicians decided to perform endoscopic per-oral pyloromyotomy. The patient was successfully treated without complications. In our experience, post-sleeve patients with gastroparesis have been safely and effectively managed with POP. Nonetheless, there is insufficient evidence in obese patients and further studies are needed. 


\section{Cholelithiasis in bariatrics}

Up to $30 \%$ of patients may develop gallstones 24 months after bariatric surgery if associated with significant weight loss. The altered anatomy following bariatric surgery poses a challenge for the management of cholelithiasis. The preferred approach in these situations is laparoscopy-assisted endoscopic retrograde cholangiopancreatography $(\mathrm{LA}-\mathrm{ERCP})^{[65]}$. However, this therapy requires multiple specialty team participation which may sometimes complicate the scenario. For this matter, a new endoscopic therapy was developed and can be performed by a single team with the use of an endoscopic ultrasound (EUS). The EUS-guided transgastric ERCP (EDGE) requires accessing the excluded stomach from the gastric pouch and creating a gastrogastric or jejunogastric fistula by using a lumen-apposing metal stent, after which a conventional ERCP is performed ${ }^{[65,66]}$. Kedia et al. ${ }^{[65]}$ compared technical and clinical outcomes of EDGE and LA-ERCP in post-RYGB patients. The success rate for therapeutic ERCP was achieved in $96.5 \%$ and 97.7\% for each group, respectively. The adverse event rate reported was $24 \%$ (7/29) for the EDGE group and $19 \%(8 / 43)$ for the LA-ERCP group. The events included perforation, pancreatitis, stent dislodgement, and bleeding; similar to those reported by Tyberg et al ${ }^{[66]}$. A multicenter experience using EDGE procedure demonstrated that it can be safely and effectively applied in postbariatric patients with biliary disease. Although both these studies have shown positive outcomes, prospective studies are needed to confirm its effectiveness and outcomes.

\section{CONCLUSION}

Minimally invasive techniques have progressed significantly over the past years. Management of obesity continues to expand, and multiple devices are now available to address these patients. Most of the endoscopic procedures mentioned have demonstrated positive outcomes with an adequate safety profile; nonetheless, there is still an opportunity for device improvement as well as physician expertise. It is of utter importance that bariatric surgeons are dexterous with the endoscope as it is a crucial tool to manage obese patients, not only as an adjuvant but also as a primary procedure. The utility of endoscopy for management in the obese population has increased substantially among our practice. We believe that the endoscopic approach in bariatrics is an appealing alternative to consider as first-line therapy. While there is still a need for long-term results and further progress, these new endoscopic techniques provide promising alternatives in the management for obesity.

\section{DECLARATIONS}

\section{Authors' contributions}

Made a substantial contribution to conception, design of the study, performed data analysis, and interpretation: Castro M, Guerron AD

\section{Availability of data and materials}

Not applicable.

\section{Financial support and sponsorship}

None.

\section{Conflicts of interest}

Dr. A. Daniel Guerron disclosed a financial relationship with Levita, Phenomix, Gore, Medtronic, and Biom'up. Dr. Castro declared that there is no conflict of interest.

\section{Ethical approval and consent to participate}

Not applicable. 


\section{Consent for publication}

Not applicable.

\section{Copyright}

(c) The Author(s) 2020.

\section{REFERENCES}

1. World Health Organization. Obesity and overweight. Available from: https://www.who.int/news-room/fact-sheets/detail/obesity-andoverweight. [Last accessed on 29 Jul 2020]

2. Guerron AD, Ortega CB, Lee HJ, Davalos G, Ingram J, et al. Asthma medication usage is significantly reduced following bariatric surgery. Surg Endosc 2019;33:1967-75.

3. ASMBS. Estimate of Bariatric Surgery Numbers, 2011-2018. Available from: https://asmbs.org/resources/estimate-of-bariatric-surgerynumbers\#. [Last accessed on 29 Jul 2020]

4. Angrisani L, Santonicola A, Iovino P, Vitiello A, Higa K, et al. IFSO worldwide survey 2016: primary, endoluminal, and revisional procedures. Obes Surg 2018;28:3783-94.

5. Fernández-Soto ML, Martín-Leyva A, González-Jiménez A, García-Rubio J, Cózar-Ibáñez A, et al. Remission of type 2 diabetes mellitus after bariatric surgery - comparison between procedures. Endokrynol Pol 2017;68:18-25.

6. Singh AK, Singh R, Kota SK. Bariatric surgery and diabetes remission: Who would have thought it? Indian J Endocrinol Metab 2015;19:563-76.

7. Ryou M, Ryan MB, Thompson CC. Current status of endoluminal bariatric procedures for primary and revision indications. Gastrointest Endosc Clin N Am 2011;21:315-33.

8. Gollisch KSC, Raddatz D. Endoscopic intragastric balloon: a gimmick or a viable option for obesity? Ann Transl Med 2020;8:S8.

9. Papademetriou M, Popov V. Intragastric balloons in clinical practice. Gastrointest Endosc Clin N Am 2017;27:245-56.

10. Ashrafian H, Monnich M, Braby TS, Smellie J, Bonanomi G, et al. Intragastric balloon outcomes in super-obesity: a 16-year city center hospital series. Surg Obes Relat Dis 2018;14:1691-9.

11. Moore RL, Seger MV, Garber SM, Smith AB, Nguyen RT, et al. Clinical safety and effectiveness of a swallowable gas-filled intragastric balloon system for weight loss: consecutively treated patients in the initial year of U.S. commercialization. Surg Obes Relat Dis 2019; $15: 417-23$

12. Yorke E, Switzer NJ, Reso A, Shi X, de Gara C, et al. Intragastric balloon for management of severe obesity: a systematic review. Obes Surg 2016;26:2248-54.

13. Sullivan S. Aspiration therapy for obesity. Gastrointest Endosc Clin N Am 2017;27:277-88.

14. Sullivan S, Edmundowicz SA, Thompson CC. Endoscopic bariatric and metabolic therapies: new and emerging technologies. Gastroenterology 2017;152:1791-801.

15. Sullivan S, Stein R, Jonnalagadda S, Mullady D, Edmundowicz S. Aspiration therapy leads to weight loss in obese subjects: a pilot study. Gastroenterology 2013;145:1245-52.e1-5.

16. Thompson CC, Abu Dayyeh BK, Kushner R, Sullivan S, Schorr AB, et al. Percutaneous gastrostomy device for the treatment of Class II and Class III obesity: results of a randomized controlled trial. Am J Gastroenterol 2017;112:447-57.

17. Nyström M, Machytka E, Norén E, Testoni PA, Janssen I, et al. Aspiration therapy as a tool to treat obesity: 1- to 4-year results in a 201-Patient Multi-Center Post-Market European Registry Study. Obes Surg 2018;28:1860-8.

18. Goyal D, Watson RR. Endoscopic bariatric therapies. Curr Gastroenterol Rep 2016;18:26.

19. Abu Dayyeh BK, Edmundowicz SA, Jonnalagadda S, Kumar N, Larsen M, et al. ASGE Bariatric Endoscopy Task Force; ASGE Technology Committee, Endoscopic bariatric therapies. Gastrointest Endosc 2015;81:1073-86.

20. Rodriguez-Grunert L, Galvao Neto MP, Alamo M, Ramos AC, Baez PB, et al. First human experience with endoscopically delivered and retrieved duodenal-jejunal bypass sleeve. Surg Obes Relat Dis 2008;4:55-9.

21. Escalona A, Pimentel F, Sharp A, Becerra P, Slako M, et al. Weight loss and metabolic improvement in morbidly obese subjects implanted for 1 year with an endoscopic duodenal-jejunal bypass liner. Ann Surg 2012;255:1080-5.

22. Sandler BJ, Rumbaut R, Swain CP, Torres G, Morales L, et al. One-year human experience with a novel endoluminal, endoscopic gastric bypass sleeve for morbid obesity. Surg Endosc 2015;29:3298-303.

23. Marinos G, Eliades C, Muthusamy R, Iki K, Kline C, et al. First clinical experience with the transpyloric shuttle (TPS(R)) device, a non-surgical endoscopic treatment for obesity: results from a 3-month and 6-month study: Abstract 2013. Available from: https://www. sages.org/meetings/annual-meeting/abstracts-archive/first-clinical-experience-with-the-transpyloric-shuttle-tpsr-device-a-non-surgicalendoscopic-treatment-for-obesity-results-from-a-3-month-and-6-month-study/. [Last accessed on 3 Aug 2020]

24. Giuliani A, Romano L, Marchese M, Necozione S, Cianca G, et al. Gastric leak after laparoscopic sleeve gastrectomy: management with endoscopic double pigtail drainage. A systematic review. Surg Obes Relat Dis 2019;15:1414-9.

25. Diaz R, Davalos G, Welsh LK, Portenier D, Guerron AD. Use of magnets in gastrointestinal surgery. Surg Endosc 2019;33:1721-30

26. Ryou M, Aihara H, Thompson CC. Minimally invasive entero-enteral dual-path bypass using self-assembling magnets. Surg Endosc 2016;30:4533-8.

27. Machytka E, Bužga M, Zonca P, Lautz DB, Ryou M, et al. Partial jejunal diversion using an incisionless magnetic anastomosis system: 
1-year interim results in patients with obesity and diabetes. Gastrointest Endosc 2017;86:904-12.

28. van Baar ACG, Holleman F, Crenier L, Haidry R, Magee C, et al. Endoscopic duodenal mucosal resurfacing for the treatment of type 2 diabetes mellitus: one year results from the first international, open-label, prospective, multicentre study. Gut 2020;69:295-303.

29. Rajagopalan H, Cherrington AD, Thompson CC, Kaplan LM, Rubino F, et al. Endoscopic duodenal mucosal resurfacing for the treatment of type 2 diabetes: 6-month interim analysis from the first-in-human proof-of-concept study. Diabetes Care 2016;39:2254-61.

30. Lopez-Nava G, Galvão MP, Bautista-Castaño I, Fernandez-Corbelle JP, Trell M, et al. Endoscopic sleeve gastroplasty for obesity treatment: two years of experience. Arq Bras Cir Dig 2017;30:18-20.

31. Barrichello S, Hourneaux de Moura DT, Hourneaux de Moura EG, Jirapinyo P, Hoff AC, et al. Endoscopic sleeve gastroplasty in the management of overweight and obesity: an international multicenter study. Gastrointest Endosc 2019;90:770-80.

32. Eslick GD. Prevalence and epidemiology of gastrointestinal symptoms among normal weight, overweight, obese and extremely obese individuals. Gastroenterol Clin North Am 2010;39:9-22.

33. Evans JA, Muthusamy VR, Acosta RD, Bruining DH, Chandrasekhara V, et al. American Societyfor Gastrointestinal Endoscopy Standards of Practice. The role of endoscopy in the bariatric surgery patient. Gastrointest Endosc 2015;81:1063-72.

34. Plevin R, Owen PJ, Marriott DB, Jones JA, Boarder MR. Role of phosphoinositide turnover and cyclic AMP accumulation in prostaglandin-stimulated noradrenaline release from cultured adrenal chromaffin cells. J Pharmacol Exp Ther 1990;252:1296-303.

35. Hernández J, Boza C. Novel treatments for complications after bariatric surgery. Ann Surg Innov Res 2016;10:3.

36. De Palma GD, Forestieri P. Role of endoscopy in the bariatric surgery of patients. World J Gastroenterol 2014;20:7777-84.

37. Ortega CB, Guerron AD, Portenier D. Endoscopic abscess septotomy: a less invasive approach for the treatment of sleeve gastrectomy leaks. J Laparoendosc Adv Surg Tech A 2018;28:859-63.

38. Guerron AD, Ortega CB, Portenier D. Endoscopic abscess septotomy for management of sleeve gastrectomy leak. Obes Surg 2017;27:2672-4.

39. Shnell M, Gluck N, Abu-Abeid S, Santo E, Fishman S. Use of endoscopic septotomy for the treatment of late staple-line leaks after laparoscopic sleeve gastrectomy. Endoscopy 2017;49:59-63.

40. Guerron AD, Ortega CB, Portenier D. Anastomotic leak following gastric bypass. In: Camacho D, Zundel N, editors. Complications in bariatric surgery. Springer; 2018. pp. 77-84.

41. Guerron AD, Rodriguez JH, Kroh M. Endoscopic management of complications: bleeding, leaks, and strictures. In: Rogula TG, Schauer PR, Fouse T, editors. Prevention and Management of complications in bariatric surgery. Oxford University; 2018.

42. Rodrigues-Pinto E, Repici A, Donatelli G, Macedo G, Devière J, et al. International multicenter expert survey on endoscopic treatment of upper gastrointestinal anastomotic leaks. Endosc Int Open 2019;7:E1671-82.

43. Mahadev S, Kumbhari V, Campos JM, Galvao Neto M, Khashab MA, et al. Endoscopic septotomy: an effective approach for internal drainage of sleeve gastrectomy-associated collections. Endoscopy 2017;49:504-8.

44. Campos JM, Ferreira FC, Teixeira AF, Lima JS, Moon RC, et al. Septotomy and balloon dilation to treat chronic leak after sleeve gastrectomy: technical principles. Obes Surg 2016;26:1992-3.

45. Diaz R, Welsh LK, Perez JE, Narvaez A, Davalos G, et al. Endoscopic septotomy as a treatment for leaks after sleeve gastrectomy: Meeting presentations: Digestive Disease Week 2019. Endosc Int Open 2020;8:E70-5.

46. Archid R, Wichmann D, Klingert W, Nadiradze G, Hönes F, et al. Endoscopic vacuum therapy for staple line leaks after sleeve gastrectomy. Obes Surg 2020;30:1310-5.

47. Rodrigues-Pinto E, Morais R, Vilas-Boas F, Pereira P, Macedo G. Role of endoscopic vacuum therapy, internal drainage, and stents for postbariatric leaks. VideoGIE 2019;4:481-5.

48. Kumar N. Endoscopic therapy for weight loss: Gastroplasty, duodenal sleeves, intragastric balloons, and aspiration. World J Gastrointest Endosc 2015;7:847-59.

49. Schulman AR, Kumar N, Thompson CC. Transoral outlet reduction: a comparison of purse-string with interrupted stitch technique. Gastrointest Endosc 2018;87:1222-8.

50. Jirapinyo P, Kumar N, AlSamman MA, Thompson CC. Five-year outcomes of transoral outlet reduction for the treatment of weight regain after Roux-en-Y gastric bypass. Gastrointest Endosc 2020;91:1067-73.

51. Horgan S, Jacobsen G, Weiss GD, Oldham JS Jr, Denk PM, et al. Incisionless revision of post-Roux-en-Y bypass stomal and pouch dilation: multicenter registry results. Surg Obes Relat Dis 2010;6:290-5.

52. Ukleja A, Afonso BB, Pimentel R, Szomstein S, Rosenthal R. Outcome of endoscopic balloon dilation of strictures after laparoscopic gastric bypass. Surg Endosc 2008;22:1746-50.

53. Parikh A, Alley JB, Peterson RM, Harnisch MC, Pfluke JM, et al. Management options for symptomatic stenosis after laparoscopic vertical sleeve gastrectomy in the morbidly obese. Surg Endosc 2012;26:738-46.

54. Bashir U, El Abiad R, Gerke H, Keech J, Parekh K, et al. Peroral endoscopic myotomy is feasible and safe in a gastric bypass population. Obes Surg 2019;29:3523-6.

55. Inoue H, Minami H, Kobayashi Y, Sato Y, Kaga M, et al. Peroral endoscopic myotomy (POEM) for esophageal achalasia. Endoscopy 2010;42:265-71.

56. Costantini A, Familiari P, Costantini M, Salvador R, Valmasoni M, et al. Poem versus laparoscopic heller myotomy in the treatment of esophageal achalasia: a case-control study from two high volume centers using the propensity score. J Gastrointest Surg 2020;24:505-15.

57. Ngamruengphong S, Inoue H, Ujiki MB, Patel LY, Bapaye A, et al. Efficacy and safety of peroral endoscopic myotomy for treatment of achalasia after failed heller myotomy. Clin Gastroenterol Hepatol 2017;15:1531-7.e3.

58. Fernandez-Ananin S, Fernández AF, Balagué C, Sacoto D, Targarona EM. What to do when Heller's myotomy fails? Pneumatic 
dilatation, laparoscopic remyotomy or peroral endoscopic myotomy: a systematic review. J Minim Access Surg 2018;14:177-84.

59. Sanaei O, Draganov P, Kunda R, Yang D, Khashab MA. Peroral endoscopic myotomy for the treatment of achalasia patients with Rouxen-Y gastric bypass anatomy. Endoscopy 2019;51:342-5.

60. Luo RB, Montalvo D, Horgan S. Peroral endoscopic myotomy after gastric bypass: An effective solution for de novo achalasia. Surg Obes Relat Dis 2017;13:e1-3.

61. Xu J, Chen T, Elkholy S, Xu M, Zhong Y, et al. Gastric peroral endoscopic myotomy (G-POEM) as a treatment for refractory gastroparesis: long-term outcomes. Can J Gastroenterol Hepatol 2018;2018:6409698.

62. Strong AT, Rodriguez J, Kroh M, Ponsky J, Cline M, et al. Safety and feasibility of per-oral pyloromyotomy as augmentative therapy after prior gastric electrical stimulation for gastroparesis. J Am Coll Surg 2019;229:589-95.

63. Rodriguez J, Strong AT, Haskins IN, Landreneau JP, Allemang MT, et al. Per-oral pyloromyotomy (POP) for medically refractory gastroparesis: short term results from the first 100 patients at a high volume center. Ann Surg 2018;268:421-30.

64. Farha J, Fayad L, Kadhim A, Şimşek C, Badurdeen DS, et al. Gastric per-oral endoscopic myotomy (G-POEM) for the treatment of gastric stenosis post-laparoscopic sleeve gastrectomy (LSG). Obes Surg 2019;29:2350-4.

65. Kedia P, Tarnasky PR, Nieto J, Steele SL, Siddiqui A, et al. EUS-directed transgastric ERCP (EDGE) versus laparoscopy-assisted ERCP (LA-ERCP) for Roux-en-Y gastric bypass (RYGB) anatomy: a multicenter early comparative experience of clinical outcomes. J Clin Gastroenterol 2019;53:304-8.

66. Tyberg A, Nieto J, Salgado S, Weaver K, Kedia P, et al. Endoscopic ultrasound (EUS)-directed transgastric endoscopic retrograde cholangiopancreatography or EUS: mid-term analysis of an emerging procedure. Clin Endosc 2017;50:185-90. 